\title{
IMPAIRED FLOW MULTI-INDEX TRANSPORTATION PROBLEM WITH AXIAL CONSTRAINTS
}

\author{
LAKSHMISREE BANDOPADHYAYA ${ }^{1}$ AND M. C. PURI ${ }^{2}$
}

(Received 13 November 1985; revised 28 July 1986)

\begin{abstract}
This paper studies the impairing of flows in multi-index transportation problem with axial constraints. For any curtailed flow, the problem is shown to be equivalent to a standard axial sum problem, whose solution can be obtained by known methods. The equivalence is established only for specially defined solutions (referred to as $M$-feasible solutions) of the standard problem. It is also proved that an optimal solution of the impaired flow problem corresponds to such an $M$-feasible solution.
\end{abstract}

\section{Introduction}

In 1973, Appa [1] studied variants of the conventional transportation problem. Another type of variant of the standard transportation problem was studied by Brigden [2] considering mixed-type constraints. Klingman and Russel [6] investigated the effect of an extra linear constraint on the transportation problem. Extension of a standard transportation problem to a three-dimensional situation with axial constraints was first examined by Schell [7]. Later on, in 1955, Corban [3] developed a solution procedure for the same problem. A three-dimensional transportation problem with planar constraints was introduced by Haley [4], who also gave a solution procedure. A solid transportation problem equivalent to Schell's "Three Axial Sums" problem was formulated by Haley [5] in another paper in 1964.

The "Three Axial Sums" problem deals with transporting of various commodities from a set of $m$ warehouses to $n$ different markets, whose total demands are

\footnotetext{
${ }^{1}$ Deshbandu College, University of Delhi, G-1356, C. R. Park, New Delhi 110019, India.

${ }^{2}$ I. I. T., Delhi, India.

(C) Copyright Australian Mathematical Society 1988, Serial-fee code 0334-2700/88 
specified. Thus if $a_{t}$ is the total availability at the $i$ th warehouse, $b_{j}$ the total demand at the $j$ th market and $e_{k}$ the total availability of the $k$ th commodity, then the multi-index transportation problem with axial constraints can be analytically stated as:

(P) Minimise $\sum_{I} \sum_{J} \sum_{K} c_{i j k} x_{i j k}$

subject to

$$
\begin{array}{ll}
\sum_{J} \sum_{K} x_{i j k}=a_{i}, & i \in I, \\
\sum_{I} \sum_{K} x_{i j k}=b_{\jmath}, & j \in J, \\
\sum_{I} \sum_{J} x_{i j k}=e_{k}, & k \in K, \\
x_{i j k} \geqslant 0, &
\end{array}
$$

where $c_{l j k}$ is the per unit cost of transporting the $k$ th product from the $i$ th warehouse to the $j$ th market and $x_{i j k}$ is the amount of the $k$ th product transported on the above route.

$I=\{1,2, \ldots, m\}$ is the index set of $m$ warehouses, $J=\{1,2, \ldots, n\}$ is the index set of $n$ markets and $K=\{1,2, \ldots, p\}$ is the index set of $p$ different products.

Obviously for any feasible solution of (P), we have $\sum_{I} a_{t}=\sum_{J} b_{J}=\Sigma_{K} e_{k}=N$ (say).

Sometimes particular situations (financial problems, outdatedness of the product, etc.) compel one to curtail the flow. At this juncture, some of the warehouses are forced to be closed down or some of them are made to operate below the original operational level, while some still continue to maintain their original supplying behaviour. The net supply of each product and the demand at each destination are also consequently affected.

The present paper discusses the above situation when the impaired flow is exactly known. A procedure is developed to determine optimal solutions of this problem which identifies the supply points selected for closure together with those preferred to operate below or at their maximum operational level. The technique also marks the affected commodities and the destinations.

\section{Theoretical development}

The flow constraint $\sum_{I} \sum_{J} \Sigma_{K} x_{i j k}=F(F<N)$ when introduced into the given system $(\mathrm{P})$, generates the following transportation problem which, mathematically 
stated is:

Minimise $\sum_{I} \sum_{J} \sum_{K} c_{i j k} x_{i j k}$

subject to

$$
\begin{aligned}
& \sum_{J} \sum_{K} x_{i j k} \leqslant a_{i}, \\
& \sum_{I} \sum_{K} x_{i j k} \leqslant b_{j}, \\
& \sum_{I} \sum_{J} x_{i j k} \leqslant e_{k}, \\
& \sum_{I} \sum_{J} \sum_{K} x_{i j k}=F, \quad(F<N) \\
& x_{i j k} \geqslant 0 .
\end{aligned}
$$

In order to solve the above problem (P-1), a related standard solid problem is formulated, with an artificial supply point, a dummy destination and an extra commodity, which is as follows:

$$
\begin{aligned}
& \text { Minimise } \sum_{I^{\prime}} \sum_{J^{\prime}} \sum_{K^{\prime}} c_{i \jmath k}^{\prime} y_{i \jmath k} \\
& \text { subject to } \\
& \qquad \sum_{J^{\prime}} \sum_{K^{\prime}} y_{i \jmath k}=a_{\imath}^{\prime}, \quad i \in I^{\prime}=I \cup\{m+1\}, \\
& \sum_{I^{\prime}} \sum_{K^{\prime}} y_{\imath \jmath k}=b_{J}^{\prime}, \quad j \in J^{\prime}=J \cup\{n+1\}, \\
& \sum_{I^{\prime}} \sum_{J^{\prime}} y_{i \jmath k}=e_{k}^{\prime}, \quad k \in K^{\prime}=K \cup\{p+1\}, \\
& y_{i \jmath k} \geqslant 0, \quad i \in I^{\prime}, j \in J^{\prime}, k \in K^{\prime},
\end{aligned}
$$

where

$$
\begin{aligned}
& a_{\imath}^{\prime}=a_{\imath}, \quad i \in I, \quad a_{m+1}^{\prime}=(N-F) / 2+\lambda, \\
& b_{J}^{\prime}=b_{\jmath}, \quad j \in J, \quad b_{n+1}^{\prime}=(N-F) / 2+\lambda, \\
& e_{k}^{\prime}=e_{k}, \quad k \in K, \quad e_{p+1}^{\prime}=(N-F) / 2+\lambda,
\end{aligned}
$$

" $\lambda$ " being an arbitrary non-negative number and

$$
\left.\begin{array}{l}
c_{1, k}^{\prime}=c_{\imath \jmath k}, \\
c_{1, p+1}^{\prime}=0, c_{i n+1 k}^{\prime}=0, c_{m+1, k}^{\prime}=0, \\
c_{i n+1 p+1}^{\prime}=M, c_{m+1 \jmath p+1}^{\prime}=M, c_{m+1 n+1 k}^{\prime}=M,
\end{array}\right\} \quad \text { for all } i \in I, j \in J, k \in K,
$$


where $M$ is a sufficiently large positive integer, much larger than each of $c_{\imath \jmath k}^{\prime}$ 's, and $c_{m+1 n+1 p+1}^{\prime}=0$.

RemarK 1. The definition of $a_{m+1}^{\prime}, b_{n+1}^{\prime}, e_{p+1}^{\prime}$ suggests that $y_{m+1 n+1 p+1}=\lambda$.

Definition. $M$-feasible solution of (P-2).

A feasible solution $\left\{y_{i j k}\right\}_{i \in I^{\prime}, j \in J^{\prime}, k \in K^{\prime}}$ of (P-2) is called an $M$-feasible solution, if $y_{i j k}=0$ whenever $c_{i j k}^{\prime}=M, i \in I^{\prime}, j \in J^{\prime}, k \in K^{\prime}$.

THEOREM 1. Any M-feasible solution of (P-2) gives a feasible solution of (P-1).

Proof. Let $\left\{y_{i j k}\right\}_{i \in I^{\prime}, j \in J^{\prime}, k \in K^{\prime}}$ be an $M$-feasible solution of (P-2). Then $\left\{x_{\imath \jmath k}=y_{\imath \jmath k}\right\}_{\imath \in I, j \in J, k \in K}$ is a feasible solution of (P-1), because $x_{\imath \jmath k} \geqslant 0$ and

$$
\begin{aligned}
\sum_{J} \sum_{K} x_{\imath \jmath k} & =\sum_{J} \sum_{K} y_{\imath \jmath k} \\
& =\sum_{J}\left(\sum_{K^{\prime}} y_{\imath \jmath k}-y_{\imath \jmath p+1}\right) \\
& =\sum_{K^{\prime}}\left(\sum_{J^{\prime}} y_{\imath \jmath k}-y_{\imath n+1 k}\right)-\left(\sum_{J^{\prime}} y_{\imath \jmath p+1}-y_{\imath n+1 p+1}\right) \\
& =a_{\imath}^{\prime}-\sum_{K^{\prime}} y_{\imath n+1 k}-\sum_{J^{\prime}} y_{\imath \jmath p+1}+y_{\imath n+1 p+1} \\
& =a_{\imath}^{\prime}-\sum_{K^{\prime}} y_{\imath n+1 k}-\sum_{J^{\prime}} y_{\imath \jmath p+1} \\
& \leqslant a_{\imath}^{\prime}=a_{\imath}, \quad \text { for } i \in I .
\end{aligned}
$$

Therefore

$$
\sum_{J} \sum_{K} x_{i j k} \leqslant a_{t}, \quad i \in I
$$

Similarly

$$
\sum_{I} \sum_{K} x_{i j k} \leqslant b_{\jmath}, \quad j \in J
$$


and

$$
\sum_{I} \sum_{J} x_{i j k} \leqslant e_{k}, \quad k \in K
$$

Again

$$
\begin{aligned}
& \sum_{I} \sum_{J} \sum_{K} x_{i \jmath k}=\sum_{I} \sum_{J} \sum_{K} y_{\imath \jmath k} \\
& =\sum_{I} \sum_{J}\left(\sum_{K^{\prime}} y_{i j k}-y_{i \jmath p+1}\right) \\
& =\sum_{K^{\prime}} \sum_{I}\left(\sum_{J^{\prime}} y_{\imath j k}-y_{i n+1 k}\right)-\sum_{I}\left(\sum_{J^{\prime}} y_{i j p+1}-y_{t n+1 p+1}\right) \\
& =\sum_{K^{\prime}} \sum_{J^{\prime}}\left(\sum_{I^{\prime}} y_{i j k}-y_{m+1, k}\right)-\sum_{K^{\prime}}\left(\sum_{I^{\prime}} y_{l n+1 k}-y_{m+1 n+1 k}\right) \\
& -\sum_{J^{\prime}}\left(\sum_{I^{\prime}} y_{l j p+1}-y_{m+1, p+1}\right)+\sum_{I} y_{l n+1} p+1 \\
& =\sum_{I^{\prime}} \sum_{J^{\prime}} \sum_{K^{\prime}} y_{1 j k}-a_{m+1}^{\prime}-b_{n+1}^{\prime}-e_{p+1}^{\prime}+2 y_{m+1 n+1 p+1} \\
& \left(\left\{y_{\imath, k}\right\}_{\imath \in I^{\prime}, j \in J^{\prime}, k \in K^{\prime}} \text { is an } M\right. \text {-feasible solution of (P-2)) } \\
& =\sum_{I^{\prime}} a_{l}^{\prime}-a_{m+1}^{\prime}-b_{n+1}^{\prime}-e_{p+1}^{\prime}+2 \lambda \\
& =\sum_{I} a_{l}-2\left(\frac{N-F}{2}+\lambda\right)+2 \lambda=N-(N-F) \\
& =F \text {. }
\end{aligned}
$$

Hence $\Sigma_{I} \Sigma_{J} \Sigma_{K} x_{t \jmath k}=F$. Thus to every $M$-feasible solution of (P-2), there corresponds a feasible solution of (P-1).

REMaRK. It is obvious that a feasible solution of (P-2), which is not an $M$-feasible solution can never correspond to a feasible solution of (P-1).

Henceforth, for following discussions, only $M$-feasible solutions of (P-2) will be considered.

THEOREM 2. The objective function value at an M-feasible solution of (P-2) is equal to that of (P-1) at its corresponding feasible solution. 
Proof. Let $\left\{x_{i j k}\right\}_{l \in I, j \in J, k \in K}$ be a feasible solution of (P-1) corresponding to an $M$-feasible solution $\left\{y_{\imath \jmath k}\right\}_{t \in I^{\prime}, j \in J^{\prime}, k \in K^{\prime}}$ of (P-2), i.e., $x_{i j k}=y_{i j k}, i \in I$, $j \in J, k \in K$. Now

$$
\begin{aligned}
& \sum_{I} \sum_{J} \sum_{K} c_{i j k} x_{i j k} \\
& =\sum_{I} \sum_{J} \sum_{K} c_{i j k}^{\prime} y_{i j k} \\
& =\sum_{I} \sum_{J}\left(\sum_{K^{\prime}} c_{i j k}^{\prime} y_{i j k}-c_{i j p+1}^{\prime} y_{i j p+1}\right) \\
& =\sum_{K^{\prime}} \sum_{I}\left(\sum_{J^{\prime}} c_{i j k}^{\prime} y_{\imath j k}-c_{i n+1 k}^{\prime} y_{\imath n+1 k}\right) \\
& \left(c_{\imath, p+1}^{\prime}=0, \text { for every } i \in I, j \in J\right) \\
& =\sum_{K^{\prime}} \sum_{J^{\prime}}\left(\sum_{I^{\prime}} c_{i \jmath k}^{\prime} y_{i \jmath k}-c_{m+1, k}^{\prime} y_{m+k \jmath k}\right) \\
& -\sum_{I}\left(\sum_{K} c_{i n+1 k}^{\prime} y_{i n+1 k}+c_{\imath n+1 p+1}^{\prime} y_{\imath n+1 p+1}\right) \\
& =\sum_{I^{\prime}} \sum_{J^{\prime}} \sum_{K^{\prime}} c_{i j k}^{\prime} y_{l, k} \quad \text { (using (1) and } M \text {-feasibility). }
\end{aligned}
$$

Hence $\sum_{I} \Sigma_{J} \Sigma_{K} c_{\imath j k} x_{\imath \jmath k}=\sum_{I^{\prime}} \Sigma_{J}, \Sigma_{K^{\prime}} c_{\imath \jmath k}^{\prime} y_{i \jmath k}$, which proves the equality of the objective functions.

THEOREM 3. If $\left\{x_{\imath, k}\right\}_{\imath \in I, J \in J, k \in K}$ is a feasible solution of (P-1), then $\left\{y_{\imath j k}\right\}_{\imath \in I^{\prime}}$, $j \in J^{\prime}, k \in K^{\prime}$ is a feasible solution of $(\mathrm{P}-2)$, where

$$
\begin{aligned}
y_{\imath \jmath k} & =x_{\imath \jmath k}, & & i \in I, j \in J, k \in K \\
& =\bar{x}_{i \jmath k}, & & (i, j, k) \in\left(I^{\prime} \times J^{\prime} \times K^{\prime}\right) \sim(I \times J \times K),
\end{aligned}
$$

$\left\{\bar{x}_{1, k}\right\}_{i \in I^{\prime}, J \in J^{\prime}, k \in K^{\prime}}$ being an $M$-feasible solution of the following "Three Axial Sums" problem:

$$
\left.\begin{array}{clc}
\text { Minimize } & \sum_{I^{\prime}} \sum_{J^{\prime}} \sum_{K^{\prime}} \bar{c}_{i \jmath k} \bar{x}_{\imath \jmath k} & \\
\text { subject to } & \\
& \sum_{J^{\prime}} \sum_{K^{\prime}} \bar{x}_{i \jmath k}=\bar{a}_{i}, \quad i \in I^{\prime}, \\
& \sum_{I^{\prime}} \sum_{K^{\prime}} \bar{x}_{i j k}=\bar{b}_{j}, \quad j \in J^{\prime}, \\
& \sum_{I^{\prime}} \sum_{J^{\prime}} \bar{x}_{i \jmath k}=\bar{e}_{k}, \quad k \in K^{\prime}, \\
& \bar{x}_{i j k} \geqslant 0,
\end{array}\right\}
$$


where

$$
\begin{aligned}
\bar{c}_{i j k}=M, \quad \begin{array}{l}
(i, j, k) \\
\text { sufficiently large positive integer } \\
\text { as defined earlier, } \\
\left.\bar{c}_{i j k}^{\prime}, \quad(i, j, k) \in\left(I^{\prime} \times J^{\prime} \times K^{\prime}\right) \sim(I \times J \times K), J^{\prime}\right)
\end{array} \\
\bar{a}_{i}=a_{\imath}-\sum_{J} \sum_{K} x_{i j k}, \quad i \in I, \\
a_{m+1}=a_{m+1}^{\prime}, \\
\bar{b}_{J}=b_{j}-\sum_{I} \sum_{K} x_{i j k}, \quad j \in J, \\
\bar{b}_{n+1}=b_{n+1}^{\prime}, \\
\bar{c}_{k}=c_{k}-\sum_{I} \sum_{J} x_{i j k}, \quad k \in K, \\
\bar{c}_{p+1}=c_{p+1}^{\prime} .
\end{aligned}
$$

Proof. Let $i \in I$. Then

$$
\begin{aligned}
& \sum_{J^{\prime}} \sum_{K^{\prime}} y_{\imath \jmath k}=\sum_{J^{\prime}}\left(\sum_{K} y_{\imath \jmath k}+y_{\imath \jmath p+1}\right) \\
& =\sum_{J} \sum_{K} y_{\imath j k}+\sum_{K} y_{\imath n+1 k}+\sum_{J^{\prime}} y_{\imath j p+1} \\
& =\sum_{J} \sum_{K} x_{i \jmath k}+\sum_{K} \bar{x}_{i n+1 k}+\sum_{J} \bar{x}_{\imath \jmath p+1} \\
& =\sum_{J} \sum_{K} x_{\imath \jmath k}+\sum_{J^{\prime}} \sum_{K^{\prime}} \bar{x}_{1, k} \quad(\text { using }(2)) \\
& =\sum_{J} \sum_{K} x_{i j k}+\bar{a}_{\imath} \\
& =\sum_{J} \sum_{K} x_{i j k}+\left(a_{\imath}-\sum_{J} \sum_{K} x_{i j k}\right) \\
& =a_{1}=a_{1}^{\prime} \text {. }
\end{aligned}
$$


Also

$$
\sum_{J^{\prime}} \sum_{K^{\prime}} y_{m+1 \jmath k}=\sum_{J^{\prime}} \sum_{K^{\prime}} \bar{x}_{m+1 j k}=\bar{a}_{m+1}=a_{m+1}^{\prime}
$$

Hence

$$
\sum_{J^{\prime}} \sum_{K^{\prime}} y_{i j k}=a_{i}^{\prime}, i \in I^{\prime}
$$

Similarly

$$
\begin{array}{ll}
\sum_{I^{\prime}} \sum_{K^{\prime}} y_{\imath \jmath k}=b_{j}^{\prime}, & j \in J^{\prime}, \\
\sum_{I^{\prime}} \sum_{J^{\prime}} y_{\imath \jmath k}=e_{k}^{\prime}, & k \in K^{\prime} .
\end{array}
$$

Obviously $y_{i j k} \geqslant 0, i \in I^{\prime}, j \in J^{\prime}, k \in K^{\prime}$. Therefore $\left\{y_{i j k}\right\}_{i \in I^{\prime}, J \in J^{\prime}, k \in K^{\prime}}$ is a feasible solution of (P-2).

REMARK 2. Theorems 1, 2, 3 prove that the optimal solution of ( $\mathrm{P}-2)$ gives an optimal solution of (P-1).

REMARK 3. As (P-2) is a standard "Three Axial Sums" problem, from Remark 2 it follows that there exists an integer optimal solution of (P-1).

\section{Numerical example}

Consider the problem (P-1) with $a_{1}=24, a_{2}=14, a_{3}=18, a_{4}=10 ; b_{1}=17$, $b_{2}=19, \quad b_{3}=21, \quad b_{4}=9 ; \quad e_{1}=17, e_{2}=31, e_{3}=18 ;$ flow constraint $\Sigma_{l} \Sigma_{J} \Sigma_{K} x_{i j k}=F=60<N=66$. The cost arranged in the form of a threedimensional matrix is as given in Table 1.

The new problem (P-2) will have then $a_{1}^{\prime}=24, a_{2}^{\prime}=14, a_{3}^{\prime}=18, a_{4}^{\prime}=10$, $a_{5}^{\prime}=(66-60) / 2+\lambda=3+\lambda ; b_{1}^{\prime}=17, b_{2}^{\prime}=19, b_{3}^{\prime}=21, b_{4}^{\prime}=9, b_{5}^{\prime}=3+\lambda$; $e_{1}^{\prime}=17, e_{2}^{\prime}=31, e_{3}^{\prime}=18, e_{4}^{\prime}=3+\lambda$, where $\lambda$ is an arbitrary non-negative number.

The cost, $c_{t j k}^{\prime}$, as defined in (P-2) is given in the form of a three-dimensional matrix in Table 2. Now solving (P-2) by the method given by Corban [3] or that given by Haley [4, 5], the optimal solution of (P-2) is depicted in circles in Table 3. From the optimal solution of (P-2), the optimal solution of (P-1) can be known and is as depicted in circles in Table 4. 
TABLE 1

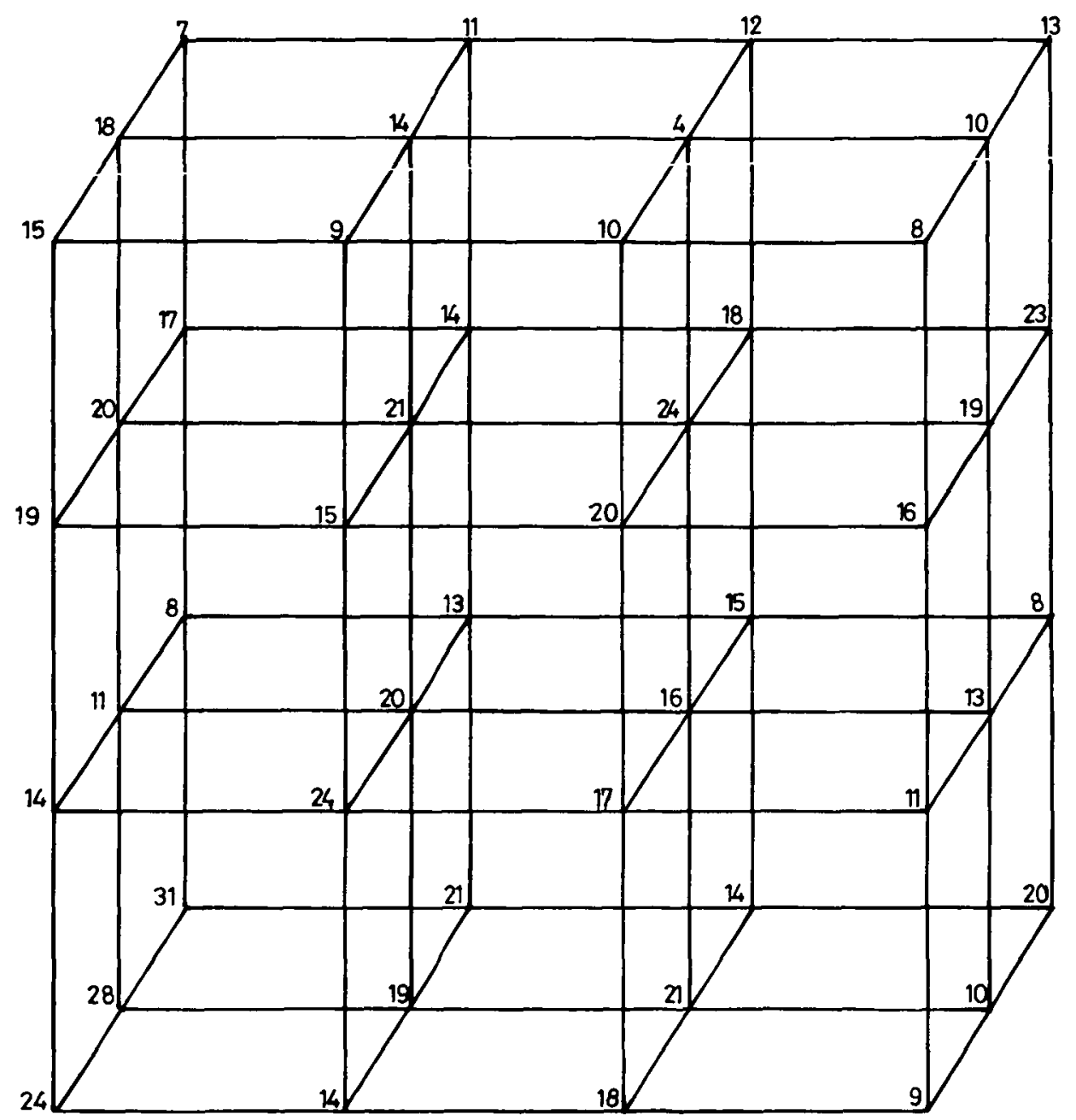


TABLE 2

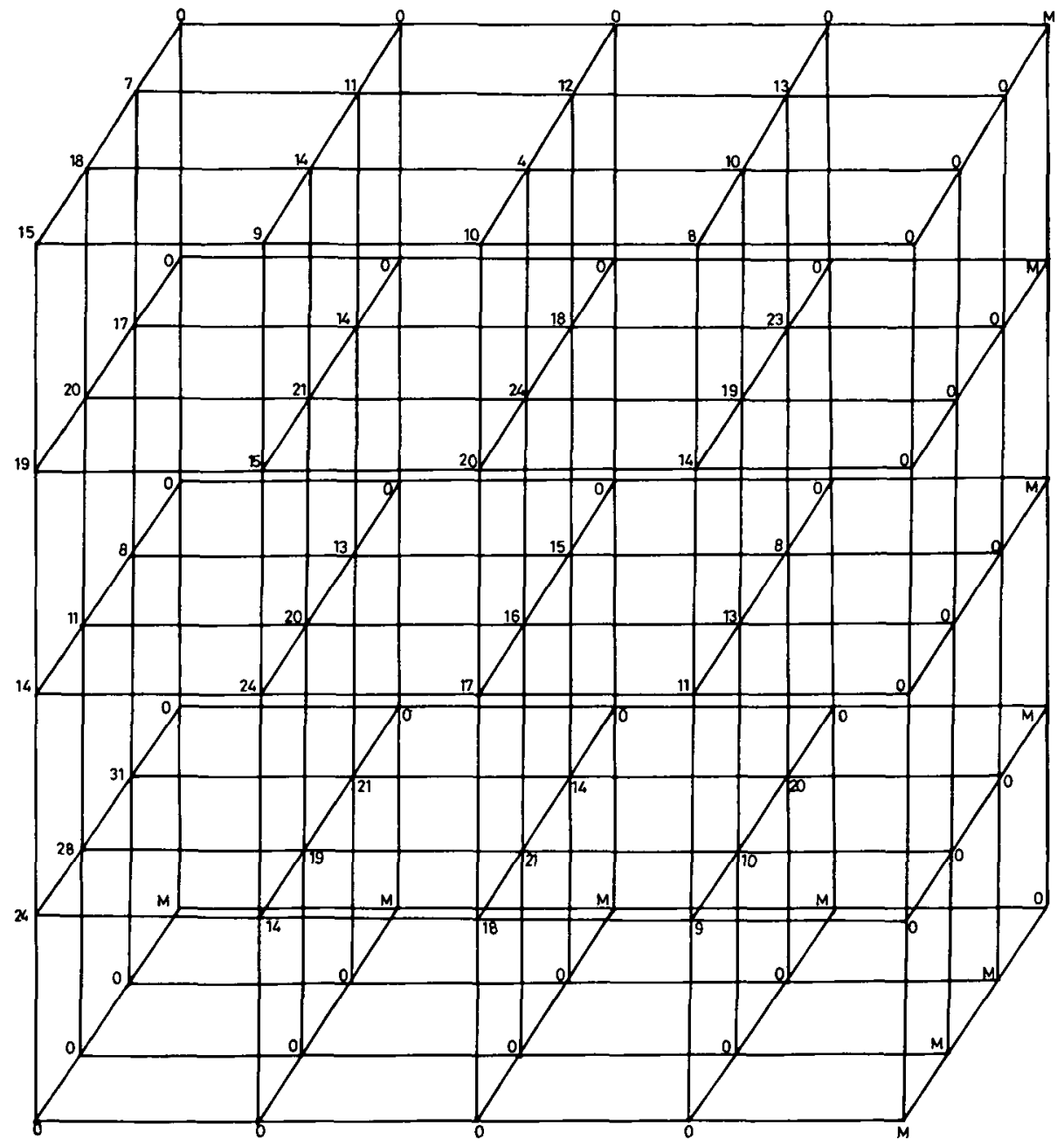


TABLE 3

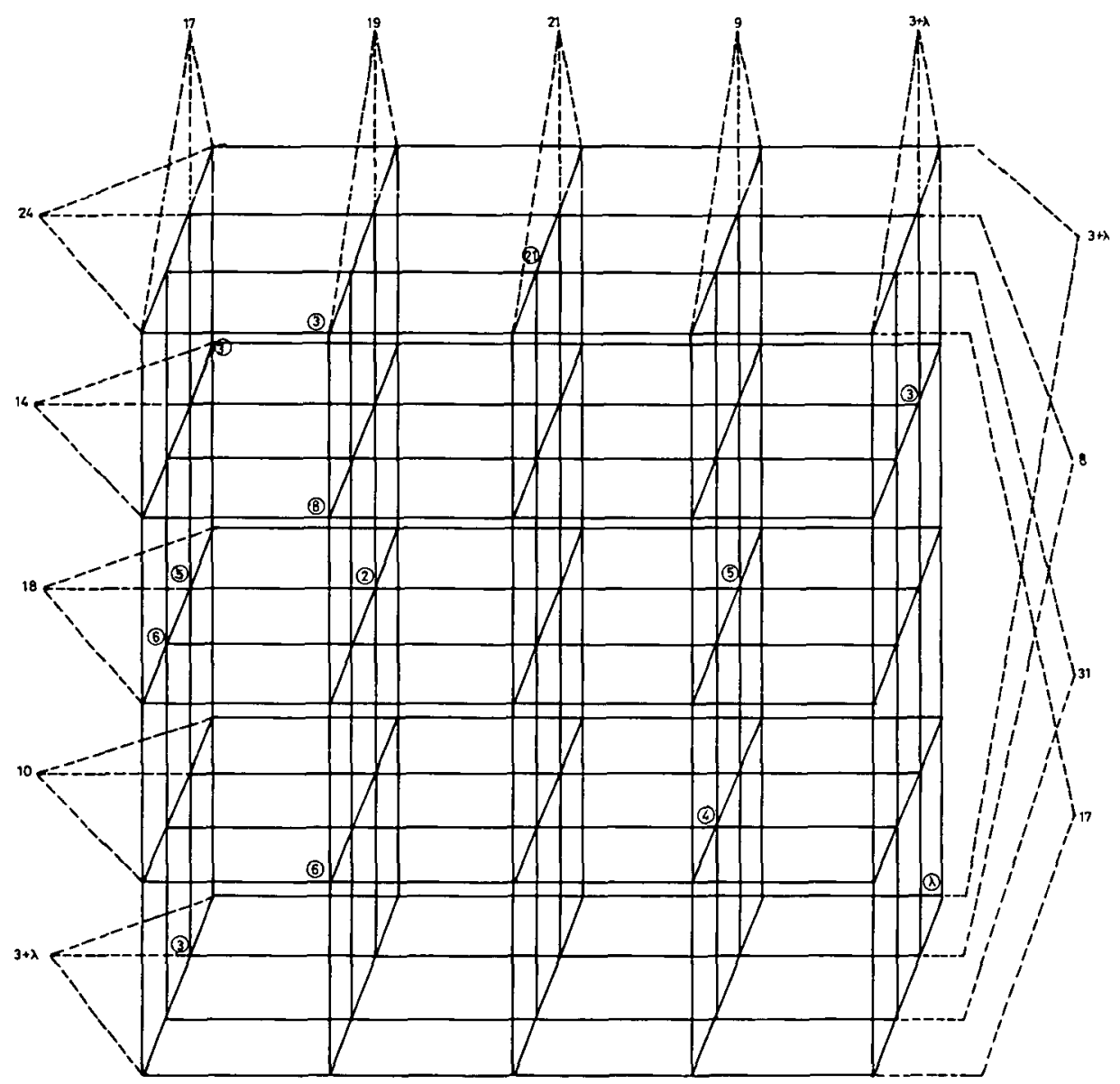


TABLE 4

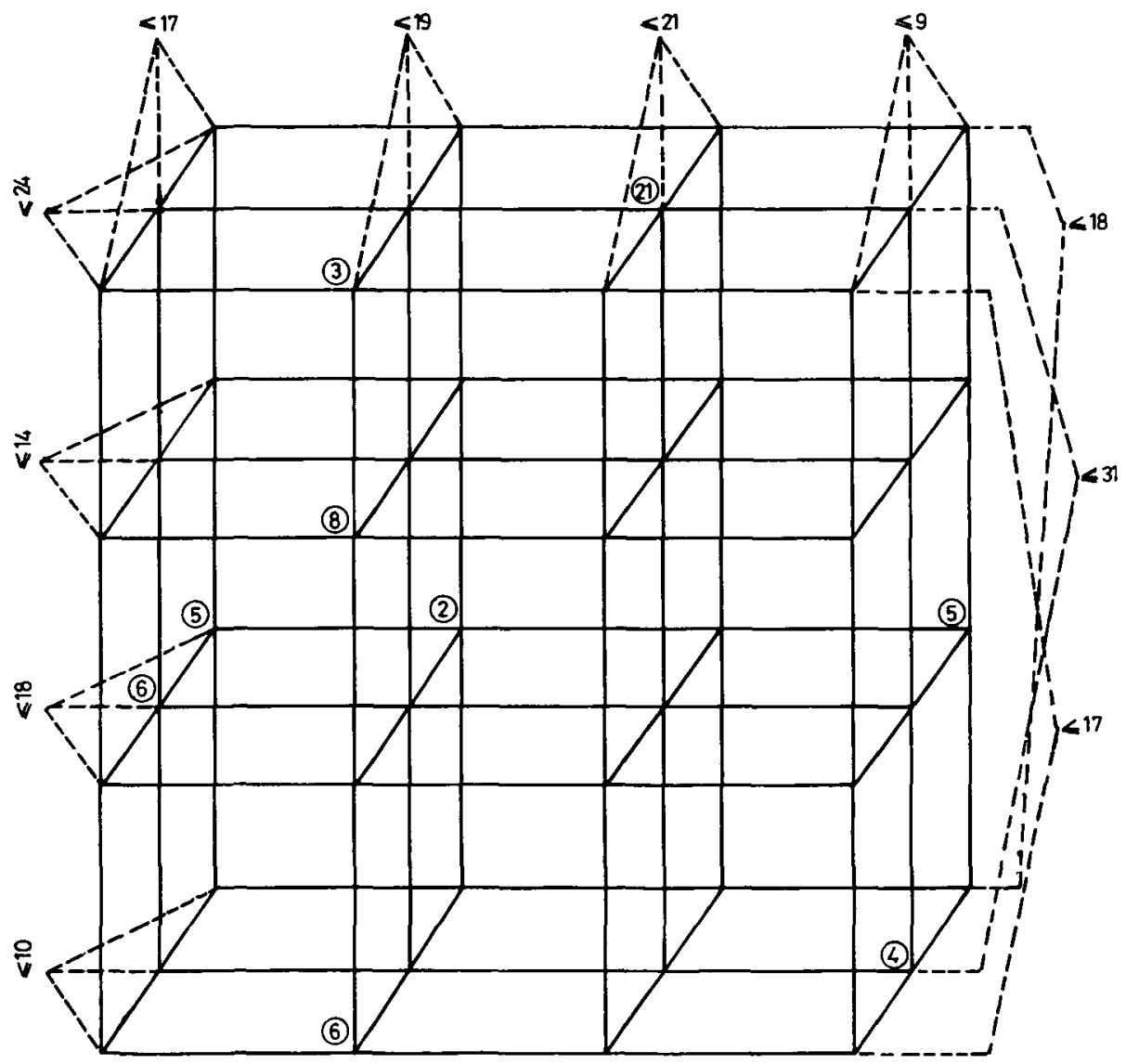




\section{Acknowledgement}

The authors are grateful to Professor R. N. Kaul, Head, Department of Mathematics, University of Delhi for his valuable suggestions.

\section{Appendix}

\section{Difference between the present paper and that of Brigden's}

Brigden [2] considered a classical transportation problem, in which the constraints are of mixed type, that is, a mixture of equality and inequality requirements and availability constraints. There is no flow constraint in his problem and thus the structure of the problem is undisturbed. He constructs an equivalent standard transportation problem and suggests special types of "shifting transformations" referring to $(\geqslant)$ constraints and "retaining transformations" dealing with $(\leqslant)$ constraints), to obtain a feasible solution of the original problem.

In the present paper, a 3-index transportation problem with axial constraints, which are very much different from the conventional single-sum constraints, is considered. The addition of a flow constraint which pertains to many practical situations further complicates the structure of the problem, resulting in problem (P-1).

Unlike Brigden's problem, (P-1) contains no " $\geqslant$ " constraints. The proposed solution procedure is therefore different from that of Brigden, in the sense that no "shifting transformations" are required and the "retaining transformations" are so defined as to take care of the additional flow constraint.

Unlike Brigden's approach, the equivalence between $M$-feasible solutions of (P-2) and feasible solution of (P-1) is established without using duality results. The proposed problem can be looked upon as a kind of variant of Schell's "Three Axial Sums" problem, whereas Brigden's is a different kind of variant of the 2-index transportation problem.

Consideration of a 3-index problem is a different and a broader aspect than the 2-index situations, in which we deal with the transportation of homogeneous goods from warehouses to markets, whereas the third index in the proposed problem takes care of various heterogeneous commodities transported from sources to destinations. An upper bound $e_{k}$ on the availability of the $k$ th commodity $(k \in K)$ in problem (P-1) in addition to upper bounds $a_{i}$ on the capacity of the $i$ th warehouse $(i \in I)$ and $b_{j}$ on the demand of the $j$ th destination $(j \in J)$ make it impossible to break the problem (P-1) in a sequence of 2-dimensional transportation problems, flow constraint further strengthening this argument. Thus 2 -indices will not suffice to deal with the proposed problem. 


\section{Characterisation of " $M$ "}

As in any feasible solution $\left\{y_{i j k}\right\}_{J^{\prime} \times J^{\prime} \times K^{\prime}}$ of (P-2),

$$
y_{\imath \jmath k} \leqslant \min _{I^{\prime}, J^{\prime}, K^{\prime}}\left(a_{\imath}^{\prime}, b_{j}^{\prime}, e_{k}^{\prime}\right) \text {, }
$$

the corresponding objective function value is at most

$$
L=\sum_{I^{\prime}} \sum_{J^{\prime}} \sum_{K^{\prime}} c_{i j k}^{\prime}\left(\min _{I^{\prime}, J^{\prime}, K^{\prime}}\left(a_{i}^{\prime}, b_{j}^{\prime}, e_{k}^{\prime}\right)\right) .
$$

As $y_{1 n+1} p+1, y_{m+1} j p+1, y_{m+1} n+1 k$ are to be nonnegative integers for all $(i, j, k) \in I \times J \times K$, it follows that a choice of $M$, sufficiently larger than $L$ would force $y_{i n+1 p+1}=y_{m+1 j p+1}=y_{m+1 n+1 k}=0$ and thus make $\left\{y_{i j k}\right\}_{I^{\prime} \times J^{\prime} \times K^{\prime}}$ $M$-feasible.

For specific purposes, $M$ can be chosen as $50 \mathrm{~L}$ or $100 \mathrm{~L}$.

\section{References}

[1] G. M. Appa, "The Transportation Problem and its Variants", Opns. Res. Quart. 24 (1973) 79-99.

[2] M. E. B. Bridgen, "A Variant of the Transportation Problem in which the Constraints are of Mixed Type", Opns. Res. Quart. 25 No. 3 (1974) 437-446.

[3] A. Corban, "Multi-dimensional Transportation Problem", Rev. Roumaine Math. Pures Appl. 9 No. 8 (1964) 721-735.

[4] K. B. Haley, “The Solid Transportation Problem”, Opns. Res. 10 (1962) 448-463.

[5] K. B. Haley, “The Multi-index Problem”, Opns. Res. Quart. 11 No. 3 (1963) 368-379.

[6] D. Klingman and R. Russel, "The Transportation Problem with Mixed Constraints", Opns. Res. Quart. 3 (1974) 447-455.

[7] E. D. Schell, "Distribution of a Product by Several Properties", Proc. 2 nd Symp. in Linear Programming, Directorate of Management Analysis, DCS/Comptroller HQUSAF (1955). 